\title{
Cushing's Disease Detected Following an Adrenal Incidentaloma
}

Gohil SG ${ }^{1}$, Kieffer $\mathrm{V}^{1}$, Levy MJ1

Department of Endocrinology, University Hospitals of Leicester NHS Trust, Leicester, LE1 5WW

Case:

- 63 year old $Q$

- Referred to Gastroenterology for change in bowel habit

- CT colonogram performed - bulky left adrenal

- Referred to Endocrinology

- Seen in the nurse led incidentaloma clinic

Investigations:
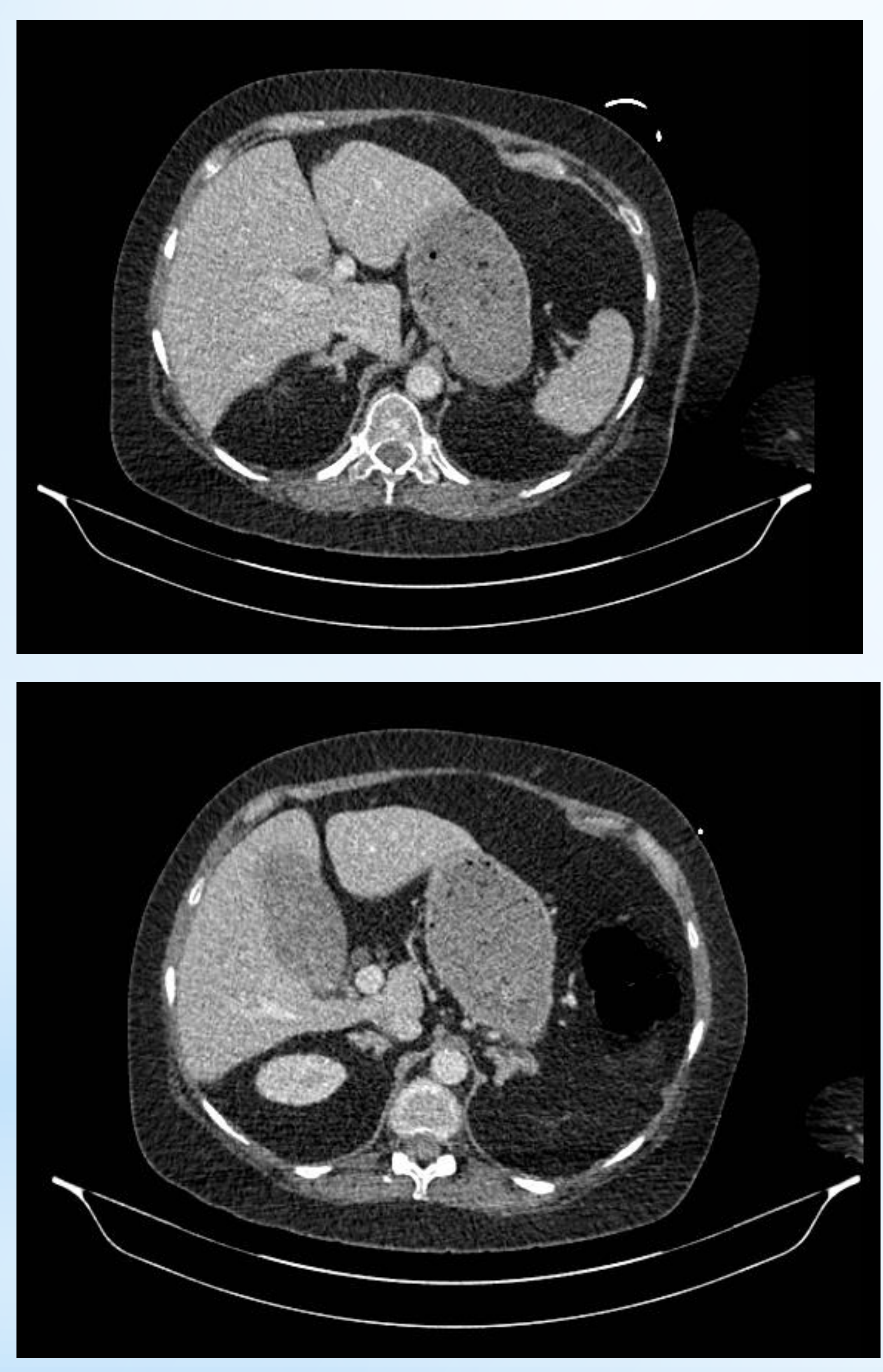

Left: Initial CT LH

colonogram

reported as

showing bulky FSH

left adrenal

measuring

$14 \mathrm{~mm}$.

Right:

Biochemistry

from nurse led

incidentaloma

clinic and

further

investigations.

\section{Discussion:}

- This patient was initially referred due to an adrenal incidenaloma.

- Clinical history and examination showed features suggestive of hypercortisolaemia.

- The patient had her initial investigations performed promptly and efficiently through a nurse-led adrenal incidentaloma clinic.

- Reviewing the images of the first CT scan again highlights bilateral bulky adrenals - confirmed with re-imaging through MRI.

- Proceeded to CRH test due to clinical suspicion of pituitary Cushing's.

- $\mathrm{CRH}$ test showed exaggerated ACTH response.

\section{Conclusion:}

Further history:

- No change in weight

- Thin arms and legs with muscle weakness

- Type 2 diabetic and hypertensive on 3 medications

- Examination - Round face, difficult getting out of chair, thin skin, bruises and dorso-cervical fat pad

- Specific nurse led adrenal incidentaloma clinics are useful to ensure patients receive appropriate initial investigations in a timely manner and helps reduce patients' visits to hospital for appointments.

- Functional adrenal tumours picked up from incidentalomas are not uncommon however functional pituitary lesions discovered following the detection of an adrenal incidentaloma is less common.

- It is important to ensure adequate investigations are performed to confirm the diagnosis before proceeding to definitive management.

- This patient has undergone a trans-spenoidal hypophasectomy and is doing well on replacement hydrocortisone. She has managed to stop all of her anti-hypertensives and her Cushing's Disease is in remission. 